\title{
Environmental Impact Evaluation of Aircraft at System-of-Systems Level
}

\author{
M.J.T. Schroijen, ${ }^{*}$ M.J.L. van Tooren $†$ M. Voskuijl $\stackrel{\ddagger}{\not}$. Curran ${ }^{\S}$ \\ Delft University of Technology, \\ Faculty of Aerospace Engineering, \\ Department of Aerospace Structures and Design Methodologies, \\ P.O.Box 50582600 GB, Delft, The Netherlands
}

\begin{abstract}
Next generations of civil transport aircraft will need to be evaluated not only against their behaviour as an aircraft system but also as a part of the larger air transport system. In addition to this are the sustainability issues related to for instance noise and emissions, represented by environmental impact. Both issues mandate the incorporation of complex stakeholder behaviour in the design and evaluation of future aircraft. A framework is proposed in extension of existing Design and Engineering Engines to perform such evaluations. For this extension a combination of agent based modelling and simulation approach, value engineering and multi-level optimization is suggested to quantitatively implement quality function deployment. As an illustration of the complexity in evaluating new aircraft concepts, the Prandtl Plane study case is used. From this study case it is found that, when evaluated using the current DEE, the Prandtl Plane shows a potential for alternative use at systems level. But no conclusion can be drawn on the environmental impact effect on the air transport system without addressing the system-of-systems considerations. This includes the unknown stakeholder response with respect to the new opportunities provided by the Prandtl Plane. One of the great challenges for future design is the increasing complexity, not only in the proposed solutions, e.g. Prandtl Plane, but also in the measures of desirability, e.g. global emissions. This requires the integrated approach addressing the complexities at both system-of-systems and system level, proposed in extension to the current design and engineering engine.
\end{abstract}

\section{Introduction}

To sustain our current wealth and health on earth, many societal and technological changes will be required. Science and technology are expected to have a major impact on the environmental impact with their contribution to the satisfaction of future human needs for food, health, transport, energy and leisure. Within the transport sector, aviation will be one of the highly challenged segments. To absorb the expected global growth in passenger and freight transport, all elements of the air transport system will need severe changes. Despite the absence of consensus on appropriate measures for a sustainable future for aviation, it is clear that environmental impact needs to be addressed at the global, i.e. system-of-systems, level. ${ }^{9}$ As a first indication, sustainability considerations on aviation should involve resource usage, noise and local and global emissions. The intention of reducing aviation environmental impact has led to the formulation of the ACARE Vision $2020^{23}$ goals as well as SESAR ${ }^{10}$ and NextGen. ${ }^{22}$ Striving to accomplish these goals should force a step change in the impact of aviation on its environment: $50 \%$ reduction in carbon dioxide $\left(\mathrm{CO}_{2}\right)$ and $80 \%$ reduction in nitrous oxides $\left(N O_{x}\right)$ emissions and elimination of noise outside the airport boundaries. For this paper we use these goals as a guide and pose that minimization of these measures provides the

*Ph.D. Student, M.J.T.Schroijen@TUDelft.nl

${ }^{\dagger}$ Professor, M.J.L.vanTooren@TUDelft.nl

¥Assistant professor, M.Voskuijl@TUDelft.nl

§Professor, R.Curran@TUDelft.nl 
desired result of reduced adverse environmental impact.

Summarizing, these measures are posed at the system-of-systems level and novel technologies are required to achieve them. ${ }^{23}$ Identifying the effect of a novel aircraft during its design on the measures is far from trivial. ${ }^{14}$ Consequently, for the age of sustainable growth ${ }^{23}$ the required improvements in quality, performance, safety, security and economy combined with the need for reduced environmental impact and increased safety level will necessitate a future aircraft system that differs as much from the current aircraft system as the current system differs from that of $1930 .{ }^{23}$ When talking about sustainable growth we assume that the only growth acceptable is asymptotic growth with the limit set according to the carrying capacity of the earth. Any other type of growth will be unsustainable by nature. In particular the unclarity about the measures most appropriate for sustainability combined with the need for novel technologies, introduces known unknowns and unknown unknowns that have to be dealt with along the way.

The goal of this paper is to identify and address the complexities present in the design for system-ofsystems level. The solutions presented should extend the current design and engineering engine to facilitate the design and evaluation of novel technologies at the system-of-systems level.

This paper addresses the framework and tools proposed for the extension of the Design and Engineering Engine (DEE). First the current DEE framework is discussed. Complexity analysis is used in order to gain a better understanding of the specific requirements for the DEE extension in order to address environmental impact in the design of novel technologies. The third section introduces the proposed elements for addressing the identified complexities. The basis for the extension is Quality Function Deployment for its focus on customer needs. To quantitatively address the intertwined stakeholder needs, which should include the environmental impact, the QFD framework is extended by an agent based modelling and simulation (ABMS) approach $^{\mathrm{a}}$, using network theory, economic principles, game theory. At the systems level these needs are translated into a mathematical formulation using value engineering. This mathematical formulation is to be addressed using the MDO approach facilitated by the DEE framework. To illustrate the complexity of the sustainability conundrum, the Prandtl Plane, as shown in Figure 1, will be used. This aircraft concept has several features not present in the current aircraft fleet. It is, however, not easy to discover to what extent these features can contribute to a sustainable future for aviation.

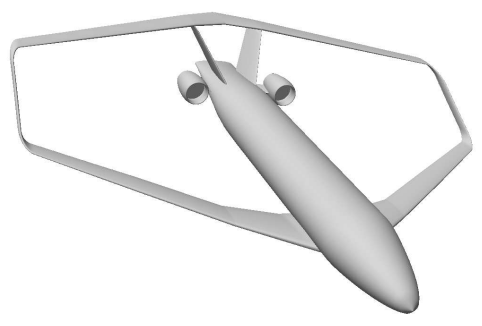

Figure 1. The Prandtl Plane.

\section{Problem analysis}

In order to support the design of future aircraft, Van Tooren and La Rocca proposed the Design and Engineering Engine (DEE) concept ${ }^{33}$ intended to automate the repetitive work and free up time and resources for creative and value adding work. The current framework is shown in Figure 2. Its main components are: 1. Requirements / design options specificator; a module that allows the user to specify objective functions, constraints and parametric product models. 2. Initiator; a module that calculates initial values for the parameters in the product model. 3. Multi-Model Generator (MMG); a KBE application that implements the parametric product model including links to the discipline tools. 4. Expert tools covering all the anal-

\footnotetext{
${ }^{a}$ not to be confused with the agents connecting the DEE components
} 


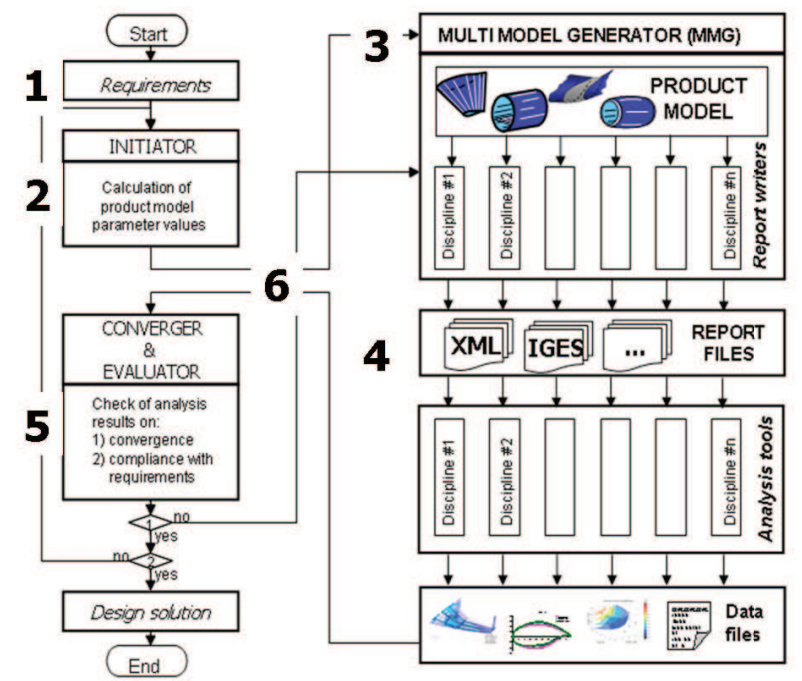

Figure 2. Existing Design and Engineering Engine framework.

yses required to derive the behaviour of the System in the different Life Cycle phases. 5. Converger and Evaluator; a module checking convergence of the behavioral models and a module with the optimizer. 6. An (agent-based) framework; the links between the different components of the DEE.This framework addresses the issues encountered in the current design for system level. In addition to this, the framework is able to find a solution within the mathematical description provided by the requirements.

As identified before, future aircraft designs have to be designed with sustainable issues in mind. The DEE has to support evaluation of the issues arising in the environmental impact assessment of novel technologies and aircraft configurations. To be able to address this issue more formally, aviation is considered as a Complex, Large-Scale, Interconnected Open Socio-Technical system. A large part of the complexity in

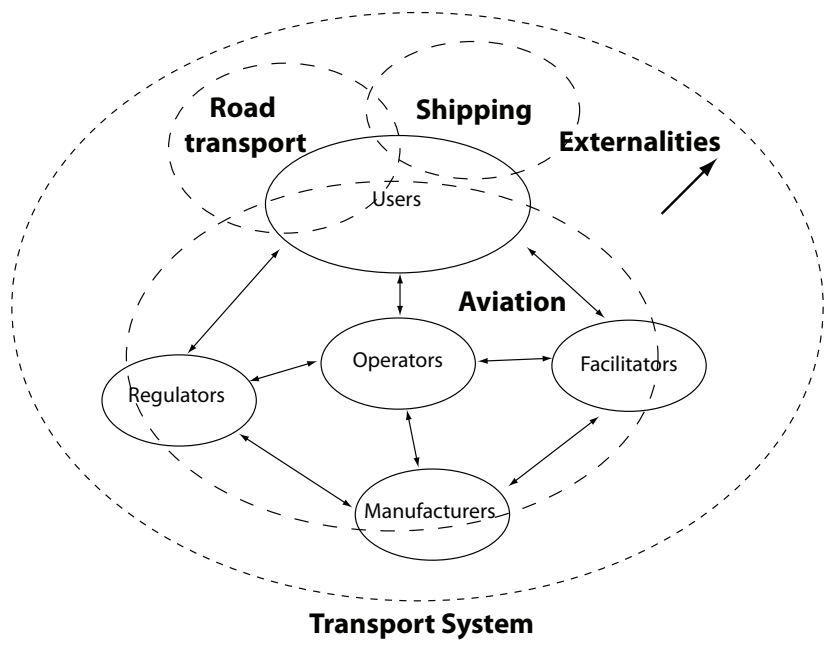

Figure 3. Schematic (subjective) overview of the stakeholders and their interactions in aviation and the broader placement of aviation in the transport system.

aviation originates from the large number of different intertwined stakeholders, all with their own goals and behaviour, resulting in many - unknown - interactions and influences. Furthermore, aviation is a part of the broader transport system. This is shown schematically in Figure 3. These interactions have a direct 
effect on the environmental impact of a novel technology. Consider as an example the introduction of a more fuel efficient aircraft, due to the decreased operation costs, the competitiveness of aviation might increase, resulting in a large share of passengers transported. Despite the decreased system level technology impact the system-of-systems level impact might be increased due to the increased use of the technology. ${ }^{25,26}$ These effects need to be considered when evaluating technology impact at the system-of-systems level, based on the system level impact. Furthermore, this complexity results in the sensitivity of the aviation system to small changes, e.g. novel technology, in particular when considering future states. ${ }^{4}$

Therefore the extension to the DEE, needs to incorporate effects from elements beyond the sphere of control (and even beyond the sphere of influence) of the stakeholder directly influenced by the technology. This represents, for example, the effect of the limited influence of the design team within the company, but also the limited company influence on its environment, e.g. passenger flows, regulations and gross national product. Furthermore, this framework should incorporate and emulate the behaviour of all stakeholders of aviation, which is far from trivial. ${ }^{14}$ As a consequence, complexities are expected at both system levels, which should be addressed appropriately. The complexities present in CLIOS, identified by Sussman, ${ }^{29,30}$ are used as a starting point. This analysis is split into considerations at the system-of-systems, i.e. aviation, and system level, i.e. aircraft. Schematically the considered complexities and the proposed DEE extension are shown in Figure 4. The tools to extend the existing DEE are described in Section III.

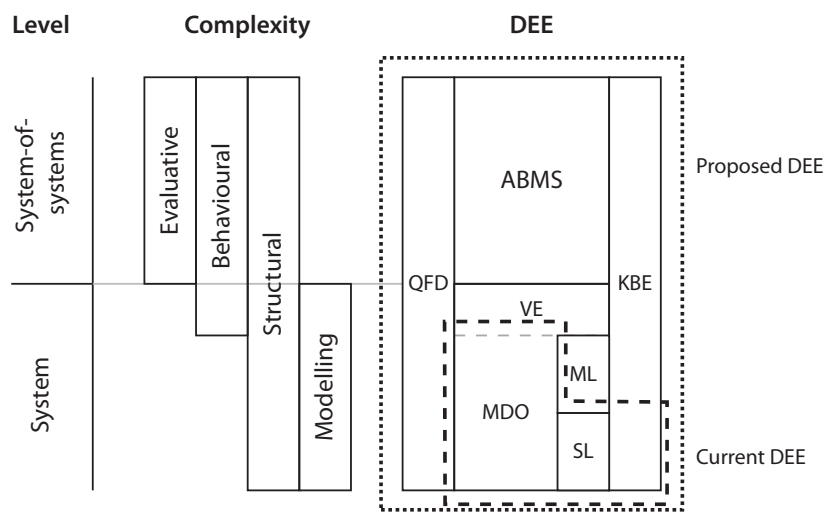

Figure 4. Relation between complexity, level and available or proposed DEE.

\section{A. System-of-systems level}

At the system-of-systems level, behavioural, structural and evaluative complexity ${ }^{30}$ are identified. Behavioural complexity arises from the fact that the exact impact of an external or internal disturbance on the system is hard to model or predict with accuracy. In particular the emerging behaviour due to the interaction of sets of components. Structural complexity is related to the number of components and the interconnections between them. Complexity rises when more elements or connections exist in a system. Evaluative complexity arises from the existence of many different stakeholders with different viewpoints about system performance - goals and behaviour - making it difficult to reach consensus on the system design. With respect to managing the physical system Sussman ${ }^{30}$ also identifies nested complexity, which is complexity arising from the introduction of complex physical systems into the existing institutional sphere. With respect to the evaluation of novel technologies this typically results in an uneven "playing field", at the disadvantage of the novel technology. This is caused by the fact that the institutional sphere is formed and optimized for existing technologies. As an example, the airline operations, the airport infrastructure and the fuel infrastructure is optimized for the tube-wing kerosene aircraft. To even this playing field, it is assumed that the point of consideration is sufficiently far ahead in time so the institutional sphere can be adapted to accommodate either technology. The resulting influence of the current institutional sphere is thus assumed negligible.

\section{B. System level}

At the system level, the system, e.g. aircraft, should be designed fulfilling the requirements and complying to the constraints. Due to the indication that conventional means are likely to be insufficient in achieving 
the sustainability criteria, ${ }^{31}$ additional complexity is introduced into the design process as well, i.e. structural complexity and modelling complexity. Structural complexity, again arises from the large number of interconnected subsystems, components and elements compromising the system and modelling complexity arises from the fact that the validity of the results, produced by first principle and high fidelity tools, remains uncertain for novel and unconventional configurations. This modelling complexity is not considered in this paper but is mentioned for completeness. Both complexities directly influence the predicted behaviour of the overall system and contribute to the behavioural complexity.

\section{Proposed framework}

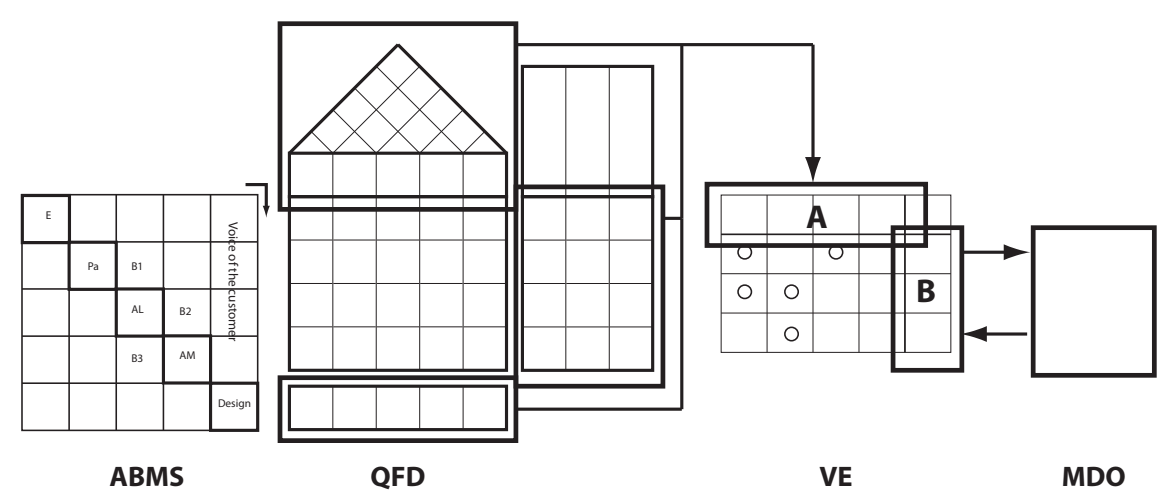

Figure 5. Quality Function Deployment as the basis, extended by Agent Based Modelling and Simulation, Value Engineering and Multidisciplinary Design Optimization.

The overall structure of the extension is shown in Figure 5. That basis is formed by Quality Function Deployment (QFD) and its visual aid the House of Quality (HOQ). ${ }^{6}$ This HOQ is extended on the left with an Agent Based Modelling and Simulation approach (ABMS) to address the structural and behavioural complexity. The right side addresses the translation from the diverse and often conflicting qualitative stakeholder needs into a mathematical formulation corresponding to a solution domain. The approach adopted for this is value engineering (VE). Finally, searching the solution domain using a multi-level multidisciplinary design optimization approach (MDO) approach.

Section A details the stakeholders which are to be considered in the design of a sustainable technology and the relation between qualitative QFD and the quantitative additions. The quantitative treatment of the intertwined stakeholders and the resulting emerging behaviour is discussed in Section B. This is followed by the VE approach in Section C. Finally the MDO approach is discussed in Section D.

\section{A. Quality Function Deployment}

From the considerations it is clear that success of a product is determined by stakeholder-product interaction, i.e. the desirability of the product for the customer. The supply side of the market, including the designers can adopt several views with respect to the demand side of the market, shown in the four different seller orientations identified by Kotler ${ }^{5}$

Prey : customers are influenced and manipulated (lowest possible quality)

Stranger : seller indifferent to customer concerns (product differentiation)

Neighbour : limited understanding of the customer (product improvement and innovation)

Friend : customer engagement (humanistic marketing)

On the demand side the behaviour on a personal level is driven by an ill-understood combination of moral, social and economic stimuli. The two sided complexity of the supply and demand interaction would be reduced if the 'friend' market view would be adopted and extended by the suppliers to truly engage with 
customers and all other stakeholders, even engaging with issues not represented by today's stakeholders. ${ }^{5}$ This in combination with a demand side that would act purely economically within strict moral and social constraints would ease the system-of-systems evaluation. This is, however, a utopian view and not the proper basis for an evaluation framework that should reflect today's reality to evaluate potential products at the system-of-systems level.

To address the environmental impact in the design of the product, it is assumed that stakeholders experiencing the externalities obtain the power to influence the design beforehand. Consequently the approach resembles the treatment of safety in design. As a consequence a narrower definition than proposed by Freeman ${ }^{19}$ is adopted for stakeholders in the aircraft design programme:

A stakeholder for an aircraft development programme (by definition) any group or individual who can affect the achievement of the programme's objectives.

This is a larger set than the set of natural stakeholders, which are normally considered in the design as identified by Mitchell. ${ }^{19}$ Mitchell ${ }^{19}$ proposed a means of identifying the stakeholder salience (i.e. relative importance), based on three stakeholder aspects; power, legitimacy and urgency. In aviation, this larger set of stakeholders can range from airport authorities and airlines to people living near an airport and is affected by the emerging behaviour. Addressing this emerging behaviour requires incorporating the interactions between stakeholders and strengthens the requirement for social network analysis ${ }^{27}$ in the elicitation of the stakeholders needs. In practice this means that not only the stakeholder needs, but also the effects of stakeholder interactions need to be considered.

The implementation of this interaction of stakeholders is treated by the agent based modelling and simulation approach (ABMS). This addresses the structural and behavioural complexity at the system-of-systems level. The translation of the qualitative need of these coupled stakeholders into a single mathematical formulation, i.e. evaluative complexity, is addressed by a Value Engineering approach. Finally the mathematical formulation is addressed using the computational design aid of the DEE, however to improve the efficiency of the optimization and address the structural and behavioural complexity, a multi-level multidisciplinary design and optimization approach is suggested.

\section{B. Agent Based Modelling and Simulation}

Due to the intertwined needs and requirements of the heterogeneous stakeholders in aviation, limiting analysis to the interaction of dyadic ties between stakeholders is insufficient ${ }^{24}$ to explain emerging behaviour. ${ }^{2,17}$ The mere implementation of a novel aircraft invalidates the ceteris paribus assumption, frequently employed in the evaluation of the value of aircraft design projects $^{18,21}$ and a broader view on design value should therefore be adopted.

In order to be able to assess the behaviour of the stakeholders quantitatively the behaviour is to be captured in computational models. For this purpose the utility concept in micro-economics is employed. On the basis of this utility, a stakeholder is assumed to base its behaviour towards a certain product or service, whereby the utility is derived from the product attributes (product properties) and the utility function (customer property). In defining the product properties the company producing/ selling the product is assumed to have influenced/ designed the product such that it maximizes its own utility function, e.g. profit maximization. Consequently these utility functions are assumed to completely determine the stakeholder behaviour. This is considered a good first approximation although in some cases it is not correct as illustrated by the concepts of bounded rationality and satisfycing. An alternative, complementary view on the identification of requirements and the objective function stems from systems engineering ${ }^{16}$ whereby the designer tries to identify the market segment (customer, technology, function) which his product is going to target. In essence this also involves identifying the particular area, country or section of the population that might buy the product and their respective motivations for doing so.

With the quantified behaviour the stakeholder behaviour can be emulated by a simulation. In order to be able to predict and evaluate the impact of the novel design on the system-of-systems level measures, the identified stakeholders and their quantified behaviours can be implemented in an agent based environment. ${ }^{20}$ This agent based environment consists of multiple independent decision making entities, called agents, which 
are allowed to interact with one another within a (directed) network. The stakeholders are considered to make their decisions, and resulting actions autonomously but are limited by the constraints set by the environment. These stakeholders can therefore be seen as agents which operate in the network, according to a set of behavioural rules. Consequently, the stakeholder network represents the environment which influences the agent's behaviour. As a result, implementing a novel aircraft into this environment, either as an agent or a new option, can have a direct or indirect influence on the behaviour of agents. That is the stakeholder/agent decision rules can be adapted due to the occurrence of the novel technology.

Therefore, the agent based environment allows the individual agents to interact with each other and react on the newly introduced novel aircraft according to their behavioural rules.

The resultant emerging behaviour can be assessed for its desirability. This requires the output of the objectives of interest, for instance $\mathrm{CO}_{2}, \mathrm{NO}_{x}$ emissions and noise levels ${ }^{\mathrm{b}}$. As a consequence, an indication of the system-of-systems impact caused by the novel technology, corrected for the stakeholder behaviour is obtained. However, these predictions are limited as the behavioural/ decision model is often based on previously portrayed behaviour and does not include the creativity and learning observed in real human/ stakeholder behaviour when confronted with novel technologies.

\section{Value engineering}

Value engineering is defined as a disciplined procedure directed toward the achievement of necessary functions for minimum cost without detriment to quality, reliability, performance or delivery. ${ }^{7}$ By cost, the monetary value of producing the product is meant and is therefore different from the cost of the environmental impact. ${ }^{8}$ For implementing the system-of-systems analysis in the design of the novel aircraft, an extended or broadened version of value engineering is proposed. This extended version should address and overcome the implicit assumptions as identified by Green ${ }^{13}$

- The function of the component being studied is an objective characteristic which remains constant over time. The problem can be identified and is well structured, although real world problems are fuzzy, dynamic and ill defined.

- Each alternative design solution is engineered to provide an equivalent level of performance, allowing an assessment on the basis of cost alone.

Concerning these two implicit assumptions Green $^{13}$ proposes two narrower definitions:

Value engineering : A systematic procedure directed toward the achievement of the required functions at least cost. Value engineering is based on the assumption that all the parties share the understanding of the functions being provided. Further, it is assumed that all feasible design alternatives provide the same level of functional performance, and can therefore be assessed on the basis of cost alone.

Value management : A structured process of dialogue and debate among a team of designers and decision makers during an intense short-term conference. The primary objective of value management is to develop a common understanding of the design problem, identify explicitly the design objectives and synthesize a group consensus about the comparative merits of alternative courses of action.

The framework used for the sustainability system-of-systems evaluation should fit somewhere in between these definitions. Cost as the sole basis of evaluation is probably too limited and artificial for environmental impact assessment, and the mere task of consulting all stakeholders and reaching consensus on the desired impact of global aviation industry is too resource intensive. Nevertheless, the principle of quantified stakeholder needs will be used as basis for environmental impact assessment.

Compound or multi-objective functions bring around the need to make trade-offs between their different elements or to unify all objective function elements into one quantity. Several methods have been devised to cope with this problem,

\footnotetext{
${ }^{\mathrm{b}}$ This analysis can be performed for various aircraft designs to be able to determine the sensitivity of the SoSs measures with respect to the aircraft performance values. As a consequence the objective function or requirements of the aircraft can be redefined to obtain a more desirable aircaft. The resulting desirability of the behaviour should be treated with caution, since the behaviour is only as reliable as the simulation environment (all important behaviours should be captured).
} 
Pareto front : Pareto fronts show those combinations of values of the elements of the multi-objective function for which no improvement can be found without deteriorating the value of other elements. It can be used as a basis for for subjective choices made by the ABS.

Utilitarianism : Bentham ${ }^{3}$ proposed that the 'good' is whatever brings the greatest happiness to the greatest number of people. This introduces the need to translate and quantify every element of the multi-objective function into a happiness value by the ABS. Known problem is that the group needs are not necessarily the summation of the individual needs. ${ }^{11,15}$

QFD : Objective functions ${ }^{6}$ are hard to define as not all desired behaviour is quantifiable. QFD (Quality function deployment) can be used to elicit the prioritization of customer needs and likings, as useful input for the ABS.

All three methods assume that a product has to be developed to fulfil someone's needs, only its parameter values are unknown and determined in an optimization process. In the evaluation framework also the rejection of a product idea, for example based on excessive externalities, should be a viable option.

\section{Multidisciplinary design optimization}

As indicated in Figure 5 and Section A and C, the identification of the design's attributes, requires analysis at the system level, the focal point of the Design and Engineering Engine. The design is driven by the requirements and should produce a feasible design, optimal for those requirements/ objective function, and constraints defined by the VE approach. In mathematical form

$$
\begin{array}{cc}
\min _{\mathbf{x}} & \phi(\mathbf{x}) \\
\text { s.t. } & \mathbf{h}(\mathbf{x})=0 \\
& \mathbf{g}(\mathbf{x}) \leq 0 \\
& \mathbf{x}_{l} \leq \mathbf{x} \leq \mathbf{x}_{\mathbf{u}}
\end{array}
$$

To efficiently analyse the proposed design, multiple analysis tools are called upon, making use of aspect decoupling. Decoupling of subsystems in their aspects or hardware introduces the need for a form of coordination. $^{32}$ To guide the optimization process in the right direction (towards a 'better' design) a multidisciplinary design optimization strategy is therefore required. For example, BLISS, Bi-level Integrated System Synthesis (BLISS) can be used to perform the analysis and optimization at subsystem level concurrently. ${ }^{28}$ BLISS provides a method to coordinate the optimization of the subsystems. The subsystems should optimize themselves with respect to a 'synthetic' objective function that measures the influence of the subsystems' design variables on the entire system objective function. The overall BLISS procedure, with a special identification of opportunities for concurrent treatment, is shown in Figure 6. BLISS employs a the global sensitivity approach for linearizing the objective. The use of this compound objective function only allows for continuous design variables. This means that for instance architectural changes cannot be evaluated in this analysis directly (e.g. the number of engines) and should be replaced by a continuous variable (e.g. total thrust), limiting the applicability to sizing studies. This non-uniqueness in formulation of the optimization problem is addressed by the VE approach as discussed in Section C.

\section{Proposed Study Case For the Evaluation Framework}

The Prandtl Plane (PP) was introduced in the introduction as the study case. This aircraft concept aims at minimization of the induced drag of aircraft. In cruise this reduces fuel consumption. The reduced drag in take-off allows the installation of less powerful engines, potentially reducing noise and emissions. The concept offers other advantages like a reduction of wing span. This would allow aircraft larger than the Airbus A380 to be designed without violating the current maximum allowable wing span. The aircraft also offers a concept called direct lift control. This allows for safer maneuvering. The minimization of induced drag is a typical systems level optimization issue. The other advantages of the concept could lead to unexpected stakeholder behaviour affecting system-of-systems level impact. This makes the study case suitable for evaluating the extended DEE. 


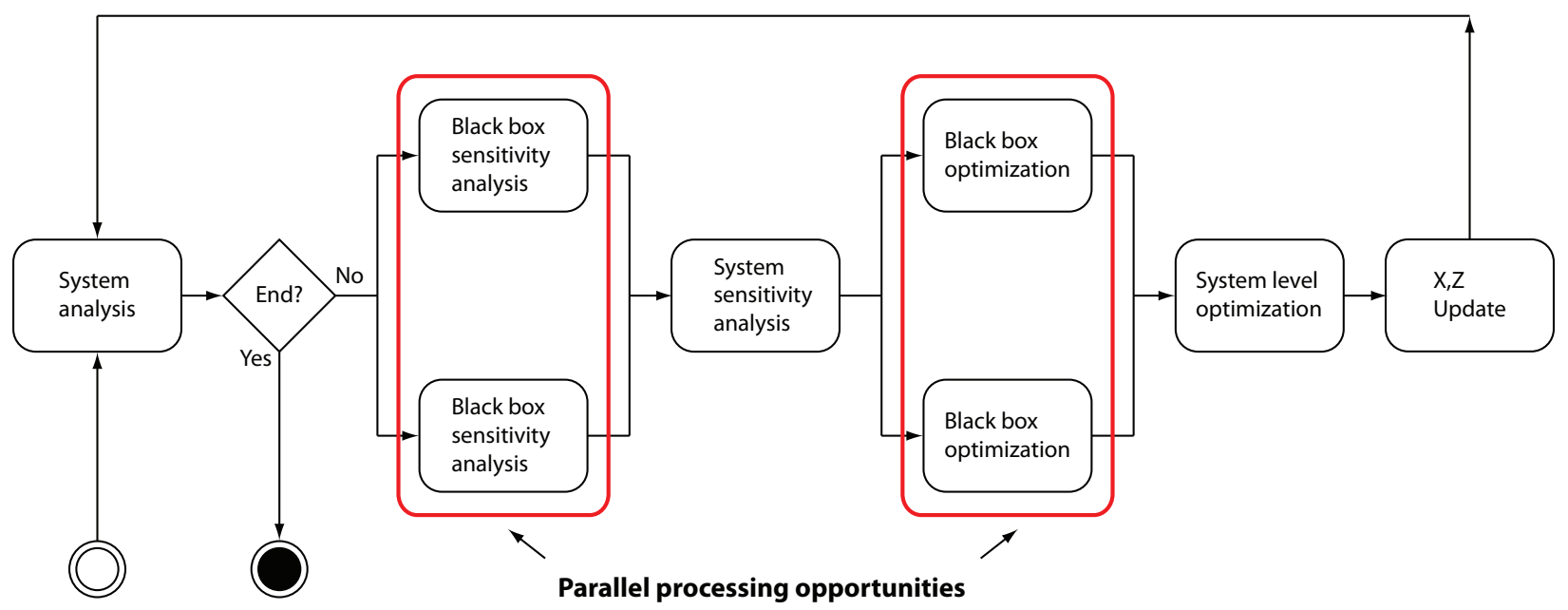

Figure 6. BLISS framework as proposed by Sobieski et al. ${ }^{28}$ The opportunities for parallel processing are marked. (Adapted from Sobieski et al. ${ }^{28}$ )

The systems optimization level has been set-up and tested already. The systems level DEE has been used to optimize the location and size of the control surfaces. ${ }^{12}$ In this study, use was made of a first order, commercial of the shelf, panel code with viscous boundary layer integration ${ }^{1}$ for the aerodynamic analysis. The aerodynamic results were used in an in-house developed flight mechanics toolbox to analyze the overall behaviour of the aircraft. An optimization study was done to minimize the total control surface area, whilst keeping an adequate level of control power in all axes. The resulting architecture is presented in Figure 7(a). It can be observed that control surfaces are present on both the front and rear wing. The inboard controls are mainly intended for longitudinal control and the outboard controls for lateral control. Each control however can have multiple functionalities. This control architecture provides two clear opportunities;

1. a pure moment can be generated by deflecting the front- and rear-wing controls in a differential manner,

2. direct lift control can be applied by deflecting the surfaces on front- and rear-wing simultaneously in one direction.

A mix of these two distinct control strategies can also be applied. If a conventional aircraft pitches up, then first a down force has to be created on the horizontal tail plane. The aircraft will initially descend slightly and once it has rotated, it will start to climb. This behaviour is typically designated as non-minimum phase behaviour. On the Prandtl Plane, this behaviour does not have to be present. The advantage of the control layout is twofold. First, airfield performance can be improved. Second, precision height control becomes significantly easier due to the direct lift capability. This is illustrated by the aircraft response to a height command as presented in Figure 7(b). One can see that the height is rapidly captured without any overshoot or non-minimum phase behaviour. The pitch attitude is continuously kept within 0.1 degrees from the trim value. This demonstrates the direct lift capability. In principle a flight control system can be designed that creates a response type somewhere between the two extremes (direct lift, pure moment) presented here. This precision control might facilitate alternative behaviour like aerial refuelling.

Next step would be to apply and evaluate the Prandtl plane concept for a typical air transport segment. The outcome on systems level should be compared to the results on system-of-systems level to evaluate the proposed framework. However, it is already clear that the substitution of a conventional aircraft with a Prandtl plane (ceteris paribus) ignores the additional potential originating from possible alternative uses. The potential for alternative take-off procedures due to the direct lift capability, or high precision altitude control. Using direct lift as an example, the complete effect of the Prandtl Plane on the environmental impact of aviation cannot be answered without taking into account possible changes in concept use, i.e. stakeholder behaviour. The evaluation of the feasibility of these alternative uses should include amongst others airport, air traffic control, airline and passenger behaviour. But also the stakeholders representing environmental 
impact like communities living near the airports. Consequently, no claim can be made with respect to the real impact at system-of-systems level (which includes environmental impact) of a novel concept without evaluating the (potential) change in stakeholder behaviour.

In conclusion, identification of alternative uses of a novel concept invalidates the ceteris paribus assumption and requires the inclusion of stakeholder behaviour in order to evaluate the concept impact. The proposed framework does not invalidate the current DEE framework but extends it. This extension increases the usability of the DEE into evaluations at the system-of-systems level, if the identified complexities are addressed appropriately.

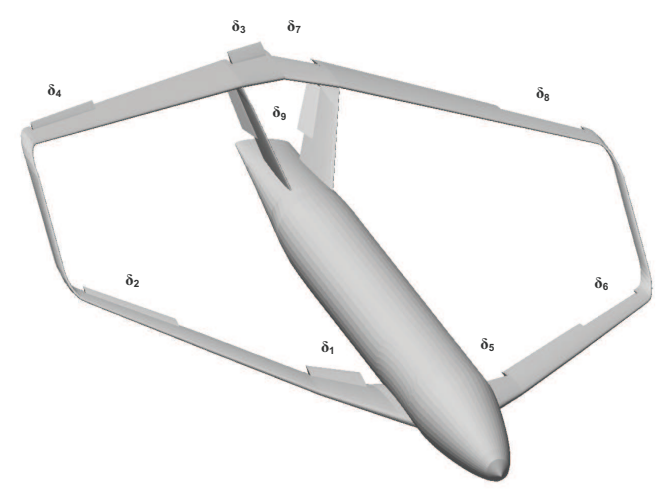

(a) Control surface layout
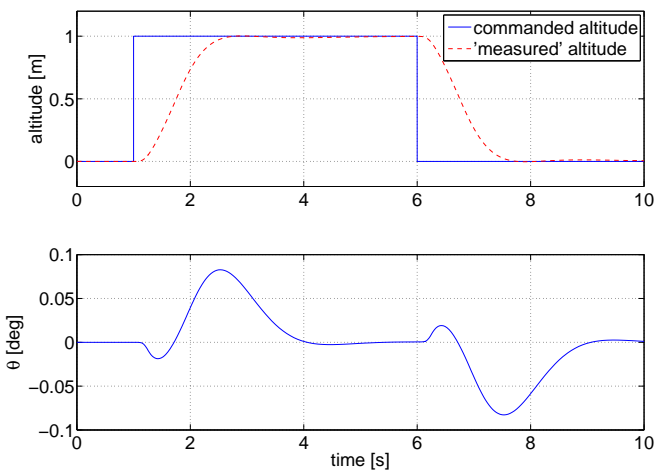

(b) Response to height command

Figure 7. Prandtl plane direct lift concept.

\section{Conclusion}

This article proposes an architecture for a framework for systems-of-systems evaluations of complex products. In addition it proposes a test case for the evaluation of the framework itself. The suggested evaluation framework is an extension of the Design and Engineering Engine that has been developed and tested for evaluation of aircraft designs on systems level.

The extended architecture defines a framework suitable for environmental impact studies in aviation. Two issues were identified, the issues of finding a performance measure for desired behaviour with respect to aviation's environmental impact and the issues arising from the fact that this performance measure is defined at systems-of-systems level (aviation) whereas the aircraft design is usually evaluated at the aircraft system level. The first issue is considered political and results in the use of the proxies like resource use, noise and global/ local air quality. The second issue, the discrepancy in level of evaluation for desired behaviour and objective function, has been evaluated from the complexity perspective identifying four complexities, evaluative, behavioural, structural and modelling. Quality function deployment (QFD) in an extended form is proposed to address these complexities both at the system-of-systems and system level. To address the behavioural and structural complexity at the system-of-systems level an agent based simulation and modelling approach is adopted to capture the interacting stakeholder behaviour. This facilitates the estimation of the novel design's impact at the system-of-systems level. To address the evaluative complexity the value engineering (VE) approach is adopted identifying and evaluating the solution space, e.g. conventional aircraft, Prandtl Plane, based on a measure of desirability, i.e. value. Furthermore, VE is used to formulate the mathematical optimization problem, i.e. objective, constraints and design vector. Finally the structural and behavioural complexity at the system level is addressed using the Bi-Level Integrated System Synthesis (BLISS) multidisciplinary design optimization (MDO) approach to find a solution to the optimization problem identified by VE.

A test case is proposed for the framework. The Prandtl plane concept has features that can be optimized and applied in different ways. The optimum on systems level could deviate considerably from the optimum on Systems of Systems level. Future work on framework and aircraft concept should elicit the practicality 
of the proposed framework. However, the need for the system of system level evaluation capabilities are supported by the findings of the Prandtl plane study case. The potential of, for instance, the possibility of direct lift in the Prandtl plane requires the evaluation of stakeholder behaviour, before conclusions can be drawn on the environmental impact.

In conclusion, the current DEE framework can be extended to address complexities arising in the evaluation at system-of-systems level. One of the consequences of this shift towards system-of-systems level evaluation is that development or design of a particular concept might be undesirable despite a (stakeholder) desirability at system level. Consequently, one of the great challenges for future design is the increasing complexity, not only in the proposed solutions, e.g. Prandtl Plane, but also in the measures of desirability, e.g. global emissions, requiring an integrated approach addressing the complexities at both system-of-systems and system level.

\section{References}

${ }^{1}$ Anonymous. VSAero, A code for calculating nonlinear aerodynamic characteristics of arbitrary configurations, User's manual Version 7.1. Analytical methods, Inc., Redmond, Washington, U.S.A., 2005.

${ }^{2}$ C. Benkard. A dynamic analysis of the market for wide-bodied commercial aircraft. Review of economic studies, 71:581$611,2004$.

${ }^{3}$ J. Bentham. An Introduction to the Principles of Morals and Legislation. Batoche books, 1781.

${ }^{4}$ P. Brooker. Civil aircraft design priorities: air quality? climate change? noise? The Aeronautical Journal, Volume 110, Number 1110(Number 2995):Pages 517-532, 2006.

${ }^{5}$ R. Buchanan and V. Margolin, editors. Discovering Design. University of Chicago press, 1990.

${ }^{6} \mathrm{~L}$. Cohen. Quality function deployment: how to make QFD work for you. Addison-Wesley, 1995.

${ }^{7}$ L. Crum. Value engineering: the organised search for value. Longman, UK, 1971.

${ }^{8}$ R. Curran, S. Raghunathan, and M. Price. Review of aerospace engineering cost modelling: the genetic causal approach. Progress in aerospace sciences, Vol. 40:pp. 487-534, 2004.

${ }^{9}$ D. DeLaurentis. Understanding transportation as system of systems design problem. In AIAA Aerospace Sciences Meeting and Exhibit 10-13 January, Reno Nevada, number 2005-0123 in AIAA, 2005.

${ }^{10}$ Eurocontrol and Europe. A vision for europe aviation. Tech. rep., EURONTROL and ACI EUROPE, 2005.

${ }^{11}$ D. Feeny, F. Berkes, B. McCay, and J. Acheson. Tragedy of the commons: twenty-two years later. Human ecology, 18(1), 1990.

${ }^{12}$ D. v. Ginneken, M. Voskuijl, M. van Tooren, and A. Frediani. Automated control surface design and sizing for the prandtl plane. In 6th AIAA Multidisciplinary Design Optimization Specialist Conference, Orlando, Florida, number 2010-3060 in AIAA, 2010.

${ }^{13} \mathrm{~S}$. Green. Beyond value engineering: smart value management for building projects. International Journal of Project Management, 12(1):49-56, 1994.

${ }^{14}$ E. Han and D. DeLaurentis. A network theory-based approach for modelling a system-of-systems. In 11th AIAA/ISSMO Multidisciplinary Analysis and optimization conference 6-8 september, Portsmouth Virginia, number 2006-6989 in AIAA, 2006.

${ }^{15} \mathrm{G}$. Hardin. The tragedy of the commons. Science, 162:1243-1248, 1968.

${ }^{16}$ E. v. Hinte and M. van Tooren. First read this. 010, 2008.

${ }^{17}$ D. Irwin and N. Pavcnik. Airbus versus boeing revisited: international competition in the aircraft market. Journal of international economics, 64:223-245, 2004.

${ }^{18} \mathrm{~J}$. Markish and K. Willcox. Value-based multidisciplinary techniques for commercial aircraft system design. AIAA Journal, 41(10), 2003.

${ }^{19} \mathrm{R}$. Mitchell. Toward a theory of stakeholder identification and salience: defining the principle of who and what really counts. Academy of management review, 22(4):853-886, 1997.

${ }^{20} \mathrm{M}$. North and C. Macal. Managing business complexity. Oxford university press, 2007.

${ }^{21} \mathrm{R}$. Peoples and K. Willcox. Value-based multidisciplinary optimization for commercial aircraft design and business risk assessment. Journal of Aircraft, 43(4), 2006.

${ }^{22}$ J. Planning and D. O. (JPDO). Next generation air transportation system integrated plan. Tech. rep., Joint Planning and Development Office, 2004.

${ }^{23}$ Report of the group of personalities. European aeronautics; a vision for 2020, 2001.

${ }^{24} \mathrm{~T}$. Rowley. moving beyond dyadic ties: a network theory of stakeholder influences. Academy of management review, 22(4):887-910, 1997.

${ }^{25}$ M. Schroijen and M. van Tooren. Decision support framework for future aircraft development programs. In 9th AIAA Aviation Technology, Integration and Operations Conference (ATIO), September 2009, Hilton Head, South Carolina, number 2009-6930 in AIAA, 2009.

${ }^{26} \mathrm{M}$. Schroijen and M. van Tooren. Environmental impact evaluation using an agent based simulation framework. In $10 t h$ AIAA Aviation Technology, Integration and Operations Conference (ATIO), September 2010, Fort Worth, Texas, number 2010-9332 in AIAA, 2010.

${ }^{27}$ J. Scott. Social network analysis: a handbook. SAGE publications, 1991.

${ }^{28}$ J. Sobieszczanski-Sobieski, J. Agte, and R. Sandusky, Jr. Bi-Level Integrated System Synthesis (BLISS). Technical Report NASA/TM-1998-208715, NASA Langley Research Center, Hampton, Virginia, August 1998. 
${ }^{29}$ J. Sussman. The new transportation faculty: The evolution to engineering systems. Transportation Quarterly, 1999.

${ }^{30} \mathrm{~J}$. Sussman. Collected views on complexity in systems, 2002.

${ }^{31} \mathrm{E}$. Torenbeek and H. Deconinck, editors. Innovative configurations and advanced concepts for future civil aircraft. Number VKI LS 2005-06 in Lecture series 2005-06. Von Karman Institute, 2006.

${ }^{32} \mathrm{~S}$. Tosserams. Distributed Optimization for Systems Design: An Augmented Lagrangian Coordination Method. PhD thesis, Eindhoven University of Technology, August 2008.

${ }^{33}$ M. van Tooren, G. L. Rocca, and T. Chiciudean. Further steps towards quantitative conceptual aircraft design, chapter 27. Springer optimization and its applications. Springer New York, 2009. 